\title{
Advanced Entry Level Protection System for Theft
}

\author{
S.ANUPRIYA ${ }^{1}$, P.ELAKYA ${ }^{2}$, B.KAMATCHI ${ }^{3}$, D.MANASA $^{4}$, G.ROHINI $^{5}$ \\ 1,2,3,4,5 Department of Information Technology, Adhiyamaan College of Engineering, Hosur, India \\ E-mail: 'bkamatchi.ace@gmail.com, ${ }^{2}$ rohinisaras@gmail.com
}

\begin{abstract}
This project is developed with aims to improve security systems available in current market. This project claims to produce a prototype camera and global system for mobile communication (GSM) interfacing system for security surveillance. This system uses sensor, namely passive infrared receiver (PIR). The PIR function is to detect changes in temperature of human in infrared radiation. This project presents a technique for human Face Recognition based on PCA algorithm (i.e., eigenface method). In this project, the microcontroller used is PIC 16, where it sends data to the computer to activate the camera when the microcontroller receives signals from PIR. MAX232 is used as an interface circuit connecting the microcontroller to the computer. To develop software for the control circuits, software MATLAB is used because the software supports microcontroller PIC 16. Once the camera is activated, it will capture images of intruder and the it will be saved and sent to the mobile phone, through commands the alarm and door lock gun will be activated. For the transmission of picture (MMS), the software used is MATLAB because they support the process of sending picture from computer to phone via GSM modem.
\end{abstract}

Keywords: Face Recognition, Camera, GSM, PIC 16 Microcontroller, Alarm, Door Lock Gun.

\section{INTRODUCTION}

Security is the degree of protection against danger, damage, loss or any criminal activity. Security is defined as a condition so that one can develop and progress freely. It is very important, because we know that crime is increasing day by day. The reason that this system is established is to create a peace of mind for people.This will help them to execute their work without any fear of their security. Through the use of security camera, users are able to monitor the situation and get timely information about persons with the fast advancing technology in recent years; camera is now widely used with the development of its content that is used in various applications. This includes visual surveillance that is in high demand in today's market. This is because of the rise in criminal rate, especially in metropolitan cities, that help boost the demand in security system that uses visual surveillance to monitor. Moreover, the area under the human's surveillance can be too wide to be monitored by only a few workers and the number of camera can exceed their monitoring ability. Security has been a major issue where crime is increasing and everybody wants to take proper measures to prevent intrusion and also provide security on detection of intrusion via MMS using GSM technology. This intelligent system needs to have the ability to scan the surrounding area and extract useful information for further consideration. For example, to detect and analyze moving activity or to identify the objects that enters the screen area. Not just limited to camera, mobile phones are also widely used. The uses of these mobile phones are not only for making and receiving calls, or for multimedia messaging services. It can also be use to send pictures that includes voice and texts to other mobile phones or email address users. This is known as multimedia messaging services (MMS). With this, mobile phone users can share their experiences and thoughts in a quick and easy way. MMS is a service that is capable to send and receive message in the form of image, graphic, video and audio in addition to text. This service plays an important part in the 3rd Generation (3G).It can be said that in terms of concept, MMS is similar to short messaging services (SMS) but with distinct difference in terms of technical for both services. After theft detection the Alarm and 
Door Lock Gun will be activated by sending commands from receiver to transmitter.

\section{RELATED WORKS}

SMS based security system was established, if any one tries to misuse the system the information will be provided as SMS to the destination. The receiver cannot identify the real-time status of the home. The signal conditioning unit is constructed with operational amplifier which acts as comparator. It received the signal from all above mentioned transducer and generates the square pulse. Then this square pulse is given to microcontroller. Here the microcontrollers PIC are flash type reprogrammable microcontroller in which we have already programmed with desired phone number.

Infrared transmitter and receiver are fixed in the entrance door. Initially the infrared transmitter transmits the rays to the receiver. If anyone crosses the rays it sends the signal to microcontroller through the signal conditioning unit. Similarly the door breaking sensor is fixed in the entrance door. If any one person breaks the door, the sensor will detect the vibration and send the signal to microcontroller through signal conditioning unit.

\section{PROPOSED SYSTEM}

In proposed system, this project provides the MMS so we can identify the intruder by sending MMS from transmitter phone to receiver phone. If anyone misuses the system, image will be captured by using web camera. The captured image will be viewed in PC by using MATLAB and the image will be recognized with the stored images, if it is matched with stored images the door will be automatically opened.

If it is not matched, the image will be transmitted to mobile phone by using mobile data cable.RS232 cable is used for interfacing the PC and PIC. The mobile phone sends this information as MMS to desired phone as per the phone number stored in the microcontroller (PIC16F877A).GSM modem is used to transmit the image from source phone to destination phone as MMS. After theft detection the Alarm and Door Lock Gun will be activated by sending commands from receiver to transmitter.

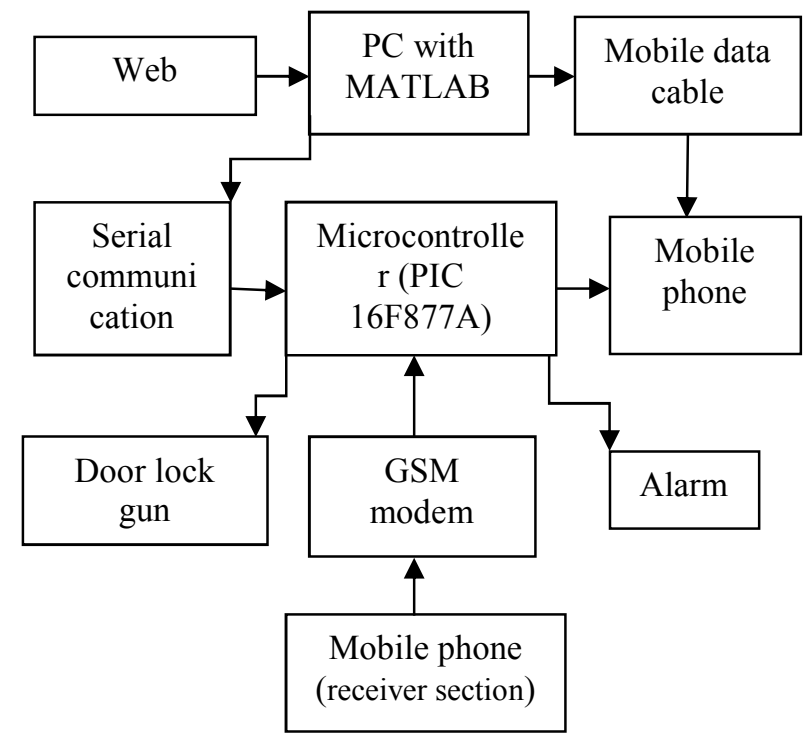

Fig. 1. System Architecture

\subsection{Face Recognition}

It includes five parts:

1. Inputimage from camera

2. Face detection

3. Pre-processing

4. Feature extraction

5. Feature matching

\subsubsection{PCA Algorithm}

PCA method (i.e., Eigen face method) is the image vector by KL transformation from highdimensional vector is converted to low dimensional vector, and the formation of low-dimensional linear vector space, that is, subspace, and then faces the projector to the low-dimensional space, with the resulting projection coefficients as the recognition feature vectors. Recognize faces, just the projection coefficient of samples to be identified in the target database sample set of projection coefficients were compared to determine what types of recently. PCA algorithm is divided into three steps: the core face database generation phase, the training phase and identification phase. This algorithm that detects human facial features likes the mouth, nose and eyes in a full frontal face image. 


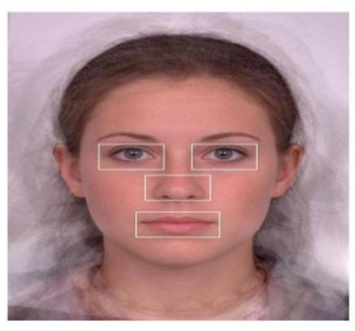

Fig. 2 Face Image

Face recognition involves two stages:

FACE DETECTION: Face detection is the stage where a photo is searched to find any face, and then image processing cleans up the facial image for easier recognition.

FACE RECOGNITION: Face recognition is the stage where that detected and processed face is compared to a database of known faces; if it is matched with stored images the door will be automatically opened. If it is not matched, the image will be transmitted to mobile phone.

\subsection{Transmitter}

If anyone misuses our system the image will be captured by using web camera. The captured image will be viewed in PC by using MATLAB and the image will be recognized with the stored images, if it is matched with stored images the door will be automatically opened.If it is not matched, it will be transmitted to mobile phone by using mobile data cable.RS232 cable is used for interfacing the PC and PIC.The mobile phone sends this information as MMS to desire phone as per the phone number stored in the microcontroller (PIC16F877A).GSM modem is used to transmit the image from source phone to destination phone as MMS.

\subsection{Receiver}

At the receiver side the transmitted image will be viewed, after theft detection the Alarm and Door Lock Gun will be activated by sending commands from receiver to transmitter.
4

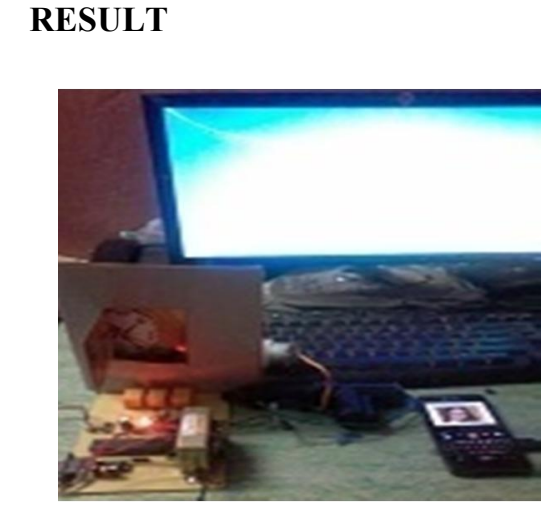

Fig. 3 Output
The main advantage of this technique is its highspeed processing capability and low computational requirements, in terms of both speed and memory utilization. The image output interfacing with mobile to mobile MMS transmission. Receiver part we can detect unknown face. That time automatic door locking system enabled. The image automatic capture using help of PIR sensor module.

\section{CONCLUSION}

This project is implemented for high level security system for theft detection. MMS in the security system can reduce break-ins incident by sending the image of offender to user in approximately $1 \mathrm{~min}$. The system was evaluated in MATLABusing an image database. This makes our system well suitedfor low-cost, real-time hardware implementation. This includes visual surveillance that is in high demand in today's market. This is because of the rise in criminal rate, especially in metropolitan cities, that help boost the demand in security system that uses visual surveillance. This project can be implemented inhigh-risk area such as banks and airports, as a standard facility in open space for the public. Now, not only high-risk areas are using it but areas such as shopping complex, offices and also industry areas are now able to have the surveillance system. 
[1] Huiping Huang, Shide Xiao, XiangyinMeng, Ying Xiong Mechanical Engineering Department Southwest Jiao tong University, Chengdu,China, "A Remote Home Security System Based on Wireless Sensor Network and GSM Technology", 2010 Second International Conference on Networks Security, Wireless Communications and Trusted Computing.

[2] NEHA SINGH1,Computer Science Department, M.I.E.R University, Lakshmangarh,Sikar District, Rajasthan 332311, India, "self initiated sms enabled home security system", Neha Singh et al. / International Journal of Engineering Science and Technology (IJEST).

[3] Amol S. Dhotre, Abhishek S. Chandurkar\& S. S. Jadhav, "Design of a GSM Cell - Phone based Vehicle Monitoring \& Theft Security System", Dept. of Electronics Engineering, GovidraoWanjari College of Engineering,Nagpur,India,Email:Amoldhotre143@gmail.com.

[4] Azid SI, Kumar S (2011),"Analysis and Performance of a Low Cost SMS Based Home Security System". Int. J. Smart Home 5(3):1524.

[5] Jin Rencheng, ZhangfTianchao, "Design and Implementation of Security and Defense System based on GSM", Computer Application,Vol.24 No.12, pp.75-77, Dec. 2004 (in Chinese). 